\title{
Perfil de mulheres climatéricas em Estratégia de Saúde da Família no interior paulista
}

\section{Health profile of climacteric women from the Family Health Strategy in the countryside of the state of São Paulo}

\author{
Victoria Marina Lima dos Santos ${ }^{1}$, Maria Isabel Sousa Vidal2 ${ }^{2}$, \\ Ana Carolina dos Santos da Silva ${ }^{3}$, Ana Maria Gelamo do Carmo ${ }^{3}$, \\ Eduardo Federighi Baisi Chagas ${ }^{4}$, Fernanda Moerbeck Cardoso Mazzetto ${ }^{5}$, \\ Maria de Lourdes da Silva Marques Ferreira ${ }^{6}$, Maria José Sanches Marin ${ }^{7}$
}

Resumo

Introdução: o climatério pode ser influenciado por mudanças psicossociais simultâneas, que associadas às alterações hormonais, como o hipoestrogenismo e a diminuição dos níveis de progesterona, podem intensificar os sintomas e agravos na mulher. Objetivo: identificar os perfis sociodemográficos, obstétricos, ginecológicos, de saúde e hábitos de vida das mulheres climatéricas atendidas na rede básica de saúde, por meio da aplicação de questionário, escalas de Hamilton de Ansiedade e Depressão, e Índice Menopausal de Kupperman e Blatt. Material e Método: estudo transversal descritivo exploratório de relato de experiência, desenvolvido por graduandas em Medicina e Enfermagem. As participantes apresentam idade entre 45 e 60 anos, atendidas em quatro unidades de Estratégia de Saúde da Família. Resultados: participaram 41 mulheres, com idade média de 52,3 anos. Prevaleceram brancas, casadas, com filhos, ensino médio completo, sem plano de saúde, escore de depressão leve, índice menopausal leve, ansiedade normal e uso regular de medicamentos. Conclusão: as mulheres climatéricas necessitam de um espaço para verbalizar seus sentimentos e dúvidas em relação ao climatério e ter acesso à escuta atenta por profissionais capacitados, visando o atendimento integral de saúde com resolutividade.

Palavras-chave: Climatério; Assistência integral à saúde; Ansiedade; Depressão; Educação em saúde.

\footnotetext{
${ }^{1}$ Graduação em Medicina pela Faculdade de Medicina de Marília (Famema), Marília, São Paulo, Brasil. E-mail: victoria.limasts@gmail.com

${ }^{2}$ Graduação em Medicina pela Faculdade de Medicina de Marília, Marília, São Paulo, Brasil.

${ }^{3}$ Graduação em Enfermagem pela Faculdade de Medicina de Marília, Marília, São Paulo, Brasil.

${ }^{4}$ Doutorado em Desenvolvimento Humano e Tecnologias pela Universidade Estadual Paulista "Júlio de Mesquita Filho" (Unesp), Rio Claro, São Paulo, Brasil. Docente do Núcleo de Ciências Biológicas e da Saúde da Universidade de Marília (Unimar), Marília, São Paulo, Brasil.

${ }^{5}$ Doutorado em Enfermagem pelo Programa de Pós-graduação em Enfermagem (Área de Cuidado em Saúde e Gestão de Sistemas) da Universidade Estadual Paulista "Júlio de Mesquita Filho" (Unesp), Botucatu, São Paulo, Brasil. Docente na Faculdade de Medicina de Marília, Marília, São Paulo, Brasil. Chefe de Disciplina de Enfermagem Gineco-obstétrica da Faculdade de Medicina de Marília, Marília, São Paulo, Brasil.

${ }^{6}$ Doutorado em Enfermagem pela Escola de Enfermagem de Ribeirão Preto da Universidade de São Paulo (EERP-USP), Ribeirão Preto, São Paulo, Brasil. Professora do Departamento de Enfermagem (Mestrado Acadêmico e Doutorado) da Universidade Estadual Paulista "Júlio de Mesquita Filho”, Botucatu, São Paulo, Brasil. Docente na Faculdade de Medicina de Marília, Marília, São Paulo, Brasil.

${ }^{7}$ Doutorado em Enfermagem Fundamental pela Universidade de São Paulo (USP), São Paulo, São Paulo, Brasil. Docente Permanente do Programa de Mestrado Profissional Ensino na Saúde e no Programa de Mestrado Acadêmico Saúde e Envelhecimento da Faculdade de Medicina de Marília, Marília, São Paulo, Brasil. Docente no Programa de Pós-graduação em Enfermagem da Universidade Estadual Paulista "Júlio de Mesquita Filho", Botucatu, São Paulo, Brasil.
} 


\begin{abstract}
Introduction: climacteric can be influenced by simultaneous psychosocial changes, which associated with hormonal changes, such as hypoestrogenism and decreased progesterone levels, can intensify symptoms and health problems in women. Objective: to identify the health profile of climacteric women in primary care regarding their obstetrics, gynecological, social, and demographic aspects, and their lifestyle as well, through a questionnaire and the application of the Hamilton Anxiety Scale, Depression Scale, and the Blatt-Kupperman Menopausal Index. Materials and Methods: it is an exploratory-descriptive cross-sectional study presented as an experience report, developed by medical and nursing students. The participants had 45 to 60 years-old and visited four units of the Family Health Strategy. Results: 41 women participated in the research, with an average age of 52.3-year-old. The majority were white, married, high school graduates, with no health insurance; had children, normal anxiety levels, mild depression, mild menopausal index, and reported regular use of medication. Conclusion: the climacteric women need some time to verbalize their feelings and doubts about the climacteric to trained professionals, aiming for a resolutive and comprehensive health care.
\end{abstract}

Keywords: Climacteric; Comprehensive health care; Anxiety; Depression; Health education.

\section{Introdução}

Segundo a Organização Mundial da Saúde (OMS), o climatério é uma etapa de transição entre o período reprodutivo e o não reprodutivo. Sua faixa etária comum abrange dos 40 aos 65 anos de idade, iniciando-se, em média, aos 47 anos, com a duração de quatro a sete anos. A irregularidade no ciclo menstrual é o seu marco inicial, e o seu término ocorre um ano após a menopausa. $\mathrm{O}$ modo de viver o climatério pode ser influenciado por mudanças psicossociais concomitantes, que associadas às modificações hormonais, como o hipoestrogenismo e a diminuição dos níveis de progesterona, podem intensificar os sintomas e problemas da mulher. Os eventos endócrinos, aliados ao aumento do Índice de Massa Corporal (IMC), contribuem de forma significativa para a elevação do risco cardiovascular, por meio do desenvolvimento de fatores de risco modificáveis. ${ }^{(1-3)}$

Também conhecida por alguns autores como transição menopáusica, essa fase compreende sintomas vasomotores, os fogachos, contribuintes para a insônia, fadiga e alterações de humor; sintomas urogenitais, como a polaciúria, noctúria, urgência miccional, ressecamento vaginal e dispareunia; ganho ponderal; e substituições teciduais mamárias. Também podem ocorrer déficits cognitivos. ${ }^{(2,4)}$
A transição menopáusica é uma questão de saúde pública, tendo em vista que a maior parte da população brasileira é composta por mulheres, principais usuárias do Sistema Único de Saúde (SUS). Em 2020, as mulheres com idade entre 40 e 59 anos constituíam $25,53 \%$ da população feminina brasileira. Além disso, elas vivem mais que os homens, considerando que a expectativa feminina de vida ao nascer, em 2018, era de 79,8 anos, enquanto a masculina, 72,74 anos. Projeções confirmam a tendência de envelhecimento populacional ao estimarem que em 2030 a população com mais de 60 anos de idade mais que duplicará, em relação aos 19,6 milhões de pessoas do mesmo grupo etário em 2010..$^{(1,5-7)}$

Contudo, as políticas e ações em saúde voltadas para a mulher climatérica são relativamente recentes no Brasil, oficializando-se em 1994, quando o Ministério da Saúde (MS) divulgou a Norma de Assistência ao Climatério, abordando determinantes biológicos, sociais e psicológicos no processo saúde-doença. Anteriormente, o enfoque da atenção à saúde feminina era dado à reprodução. ${ }^{(1)}$

Dessa maneira, considerando a diversidade de mudanças que a mulher pode vivenciar na transição menopáusica e o reconhecimento do cuidado à saúde nesta fase da vida como questão de saúde pública, torna-se importante conhecer melhor a 
população feminina climatérica usuária da rede básica de saúde. A identificação do seu perfil social, sinais, sintomas e outros problemas mais comuns experienciados no climatério fornecem informações relevantes para as equipes básicas de saúde. A partir dos resultados do presente estudo, as equipes poderão elaborar estratégias que facilitem a criação de vínculo dessas usuárias com as unidades de saúde, e propiciem a equidade, integralidade e maior resolubilidade no atendimento, melhorando a qualidade de vida dessas mulheres. O estudo, ainda, pode promover uma aproximação entre a comunidade acadêmica e a comunidade local, além de proporcionar motivação para estudos posteriores.

Portanto, objetivou-se identificar os perfis sociodemográficos, obstétricos, ginecológicos, de saúde e hábitos de vida das mulheres climatéricas atendidas na rede básica de saúde, por meio da aplicação de questionário, escalas de Hamilton de Ansiedade e Depressão, e Índice Menopausal de Kupperman e Blatt.

\section{Material e Método}

Trata-se de um estudo transversal descritivo exploratório. A coleta de dados foi realizada no período de março de 2016 a março de 2017, durante o qual foram aplicadas escalas de ansiedade, depressão e índice menopausal a 41 mulheres, de 45 a 60 anos, atendidas em quatro unidades de Estratégia de Saúde da Família (ESF), escolhidas aleatoriamente das zonas Leste, Norte e Sul de um município do interior do estado de São Paulo, Brasil.

A análise estatística do dado contínuo idade foi realizada medindo-se a tendência central por meio da média aritmética. Os escores da Hamilton Anxiety Scale (HAS), Hamilton Depression Scale (HDS) e do Índice Menopausal de Kupperman e Blatt (IMKB) foram descritos como dados ordinais. O IMKB, um instrumento validado no Brasil e utilizado para especificar e classificar os sintomas do climatério, envolve 11 sintomas-queixas (insônia, vasomotores, parestesias, nervosismo, melancolia, vertigem, artralgia/mialgia, cefaleia, palpitações, fadiga e formigamento). Para cada sintoma é estabelecido um peso (escore) diferente, que varia de leve (resultado menor ou igual a 19); moderado (se for entre 20 e 25 ) e intenso (se maior que 35), propondo maior aproximação da menopausa, período caracterizado pela ausência da menstruação confirmada em 12 meses. Para obter o total de pontos, basta somar os escores e quanto maior for a pontuação obtida, mais intensa será a sintomatologia da mulher. ${ }^{(8)}$

Para captação dos dados foram utilizados instrumentos específicos. A Escala de Ansiedade de Hamilton (HAS) é um instrumento de heteroavaliação, desenvolvido originalmente por Max Hamilton, em 1959, para medir os componentes psíquicos e somáticos da ansiedade. Consiste em 14 itens organizados numa escala com cinco pontos (de não presente a muito grave). Nesta escala muitos aspectos somáticos são considerados, tais como cardiovasculares, gastrointestinais, respiratórios e geniturinários. Esta escala pode ser subdividida em duas escalas e dois subescores: ansiedade psíquica e ansiedade somática. $\mathrm{O}$ escore total é obtido pela soma dos valores (graus) atribuídos em todos os 14 itens da escala, cujo resultado varia de 0 a 56 . Os graus de ansiedade, segundo a Escala de Ansiedade de Hamilton, são: Nenhum $=0$; Leve $=1$; Médio $=$ 2 ; Forte $=3$; Máximo $=4$. A soma dos escores obtidos em cada item resulta em um escore total, que varia de 0 a 56 . Esse escore deve ser classificado de acordo com os intervalos a seguir: 0 (zero) caracteriza ausência de ansiedade; 1 a 17 pontos: ansiedade leve; 18 a 24 pontos: ansiedade moderada; 25 a 56 pontos: ansiedade severa ou intensa. ${ }^{(9)}$

Os dados nominais estado civil, cor, filhos, uso regular de medicamentos, plano de saúde e sintomas climatéricos foram descritos da mesma forma que os dados ordinais das escalas e índice menopausal, ou seja, segundo valores absolutos e distribuição em porcentagem por categoria. ${ }^{(10)}$

$\mathrm{O}$ delineamento do estudo foi transversal. A população-alvo foram mulheres climatéricas entre 
45 a 60 anos, usuárias da rede básica de saúde de um município do interior de São Paulo. Foram realizadas as caracterizações sociodemográfica, de hábitos de vida e de saúde. Foram utilizados os seguintes instrumentos: Escala de Avaliação de Ansiedade de Hamilton, cuja pontuação varia de 0 a 56 pontos; Escala de Depressão de Hamilton (HAM-D), de 0 a 54 pontos; e IMKB (Índice Menopausal de Kupperman e Blatt), de 0 a 51 pontos. Foram convidadas a participar das entrevistas as mulheres que procuraram os serviços das ESFs, com a aplicação dos instrumentos até atingir o critério de saturação, baseado na disponibilidade das participantes.

Em relação à análise estatística, as variáveis quantitativas foram descritas pela média e desviopadrão (DP). As variáveis qualitativas foram descritas pela distribuição de frequências relativa (\%) e absoluta (N). Para analisar as diferenças na distribuição de frequências das variáveis qualitativas foi realizado o teste do Qui-quadrado para proporção. Para analisar a relação entre as variáveis qualitativas foi realizado o teste do Qui-quadrado para associação. Os dados foram analisados no software IBM $^{\circledR}$ SPSS $^{\circledR}$ versão 19.0 for Windows ${ }^{\circledR}$. O nível de significância adotado foi de $5 \%(\mathrm{p} \leq 0,05)$.

$\mathrm{O}$ projeto de pesquisa foi desenvolvido a partir da tese de doutorado intitulada "Ansiedade e depressão em mulheres climatéricas mães e em mulheres que não tiveram filhos atendidas na Rede Básica de Atenção à Saúde do Município de Marília", ${ }^{(11)}$ na qual as estudantes atuaram como colaboradoras ao participarem da coleta de dados, por meio da aplicação de questionário e das escalas. Sob a orientação da autora da tese, esses dados foram utilizados para desenvolver o presente projeto, contribuindo para a iniciação científica das pesquisadoras em formação. A tese foi submetida ao Comitê de Ética em Pesquisa (CEP) da Universidade Estadual Paulista "Júlio de Mesquita Filho" (Unesp), Campus de Botucatu, no estado de São Paulo, Brasil, sob n ${ }^{\circ}$ do parecer: $1.031 .180 \mathrm{em}$ 22/04/2015 e no do CAAE: 43082915.7.0000.5411, sob a resolução/CNS n ${ }^{\circ}$ 510/2016.

\section{Resultados}

Participaram 41 mulheres, na faixa etária de 45 a 60 anos com média e desvio-padrão de 52,9 \pm 4,7 anos. Houve predomínios da cor autodeclarada branca, do estado civil casada, com filhos, de escolaridade nível ensino médio completo, escore de depressão leve, índice menopausal leve, ansiedade normal e uso regular de medicamentos, residentes na área de abrangência de quatro unidades de ESF do interior paulista. No presente estudo, predominaram as participantes que faziam uso regular de medicamentos $(85,2 \%$, com $\mathrm{p}<0,001)$, bem como os escores de depressão leve (51,9\%), ansiedade normal $(53,7 \%)$ e sintomas climatéricos leves $(51,9 \%)(p<0,001)$. A maioria das participantes completou o ensino médio (33,3\%) e não completou o ensino fundamental $(29,6 \%)$, com $\mathrm{p}<0,001$, conforme apresentado na Tabela 1 .

Não houve associação significativa entre os sintomas do climatério e as características da amostra, por isso não apresentaremos essa tabela. O mesmo ocorreu quanto à faixa etária, cor, estado civil, ter ou não filhos, ter ou não plano de saúde e uso regular de medicamentos em relação aos escores de ansiedade, por isso essas características não foram apresentadas na Tabela 2 . Também não houve associação significativa da faixa etária, cor, estado civil, ter ou não filhos, ter ou não plano de saúde em relação aos escores de depressão, o que justifica sua exclusão da Tabela 3.

Embora houvesse predominância de mulheres climatéricas sem plano de saúde $(65,85 \%)$ e com uso regular de medicamentos, a maioria relatou ter boas condições de vida e acesso ao serviço público de saúde, no qual são geralmente atendidas quanto às suas queixas biológicas, sendo o fogacho a mais comum, além da irritabilidade e da ansiedade. Comumente, também frequentam a unidade de saúde (ESF) para realizar consultas de prevenção contra câncer de colo do útero e câncer de mama, no entanto, esses momentos têm se mostrado insuficientes para sanarem suas dúvidas sobre o climatério. 
Tabela 1 - Caracterização das unidades de saúde, dados sociodemográficos, uso de medicamentos, sintomas da menopausa, ansiedade e depressão das participantes. Marília, São Paulo. 2016.

\begin{tabular}{|c|c|c|c|c|}
\hline & & $\mathbf{N}$ & $\%$ & p-valor \\
\hline \multirow{4}{*}{ Unidade de ESF } & Novo Horizonte & 16 & 29,6 & \multirow{4}{*}{0,304} \\
\hline & Aeroporto & 8 & 14,8 & \\
\hline & Jardim Renata & 13 & 24,1 & \\
\hline & Três Lagos & 17 & 31,5 & \\
\hline \multirow{3}{*}{ Faixa etária } & $45-49$ & 14 & 25,9 & \multirow{3}{*}{0,069} \\
\hline & $50-54$ & 14 & 25,9 & \\
\hline & $55-60$ & 26 & 48,1 & \\
\hline \multirow{4}{*}{ Cor } & Branca & 36 & 66,7 & \multirow{4}{*}{$<0,001 *$} \\
\hline & Parda & 11 & 20,4 & \\
\hline & Preta & 5 & 9,3 & \\
\hline & Outra & 2 & 3,7 & \\
\hline \multirow{6}{*}{ Escolaridade } & Fundamental incompleto & 16 & 29,6 & \multirow{6}{*}{$<0,001 *$} \\
\hline & Fundamental completo & 8 & 14,8 & \\
\hline & Médio incompleto & 4 & 7,4 & \\
\hline & Médio completo & 18 & 33,3 & \\
\hline & Superior incompleto & 1 & 1,9 & \\
\hline & Superior completo & 7 & 13,0 & \\
\hline \multirow{5}{*}{ Estado civil } & Casada & 34 & 63,0 & \multirow{5}{*}{$<0,001^{*}$} \\
\hline & Solteira & 8 & 14,8 & \\
\hline & Amasiada & 1 & 1,9 & \\
\hline & Divorciada & 5 & 9,3 & \\
\hline & Outro & 6 & 11,1 & \\
\hline \multirow{2}{*}{ Filhos } & Não & 11 & 20,4 & \multirow{2}{*}{$<0,001 *$} \\
\hline & Sim & 43 & 79,6 & \\
\hline \multirow{2}{*}{ Plano de saúde } & Não & 34 & 63,0 & \multirow{2}{*}{0,057} \\
\hline & Sim & 20 & 37,0 & \\
\hline \multirow{2}{*}{ Medicamentos } & Não & 8 & 14,8 & \multirow{2}{*}{$<0,001^{*}$} \\
\hline & Sim & 46 & 85,2 & \\
\hline \multirow{3}{*}{ Sintomas da menopausa } & Leve & 28 & 51,9 & \multirow{3}{*}{$<0,001^{*}$} \\
\hline & Moderado & 23 & 42,6 & \\
\hline & Forte & 3 & 5,6 & \\
\hline \multirow{4}{*}{ Ansiedade } & Normal & 29 & 53,7 & \multirow{4}{*}{$<0,001^{*}$} \\
\hline & Leve & 11 & 20,4 & \\
\hline & Moderada & 7 & 13,0 & \\
\hline & Severa & 7 & 13,0 & \\
\hline \multirow{4}{*}{ Depressão } & Normal & 9 & 16,7 & \multirow{4}{*}{$<0,001 *$} \\
\hline & Leve & 28 & 51,9 & \\
\hline & Moderada & 8 & 14,8 & \\
\hline & Severa & 9 & 16,7 & \\
\hline
\end{tabular}

Fonte: dados da pesquisa.

Nota: * indica diferença significativa entre as categorias pelo teste do Qui-quadrado para p-valor $\leq 0,05$. 
Grande parte das participantes com sintomas climatéricos leves $(75,0 \%)$ apresentou ansiedade normal e a maioria daquelas com sintomas fortes do climatério $(66,7 \%)$ obteve escore de ansiedade severa $(\mathrm{p}<0,001)$, mostrando haver uma relação entre os escores de ansiedade e de sintomas climatéricos.
Houve ainda associação entre depressão e sintomas climatéricos, quando a maioria absoluta (100,0\%) das mulheres com sintomas fortes apresentou depressão severa, enquanto mais da metade $(60,3 \%)$ daquelas com sintomas moderados apresentou depressão leve $(p<0,001)$, conforme Tabela 2 .

Tabela 2 - Análise da associação da distribuição de frequências absoluta (N) e relativa (\%) dos sintomas da menopausa com ansiedade e depressão. Marília, São Paulo. 2016.

\begin{tabular}{|c|c|c|c|c|c|c|}
\hline & & \multicolumn{5}{|c|}{ Sintomas da menopausa } \\
\hline & & Frequência & Leve & Moderado & Forte & p-valor \\
\hline \multirow[t]{8}{*}{ Ansiedade } & Normal & $\mathrm{N}$ & 21 & 8 & 0 & $<0,001 *$ \\
\hline & & $\%$ & 75,00 & 34,80 & 0,00 & \\
\hline & Leve & $\mathrm{N}$ & 4 & 7 & 0 & \\
\hline & & $\%$ & 14,30 & 30,40 & 0,00 & \\
\hline & Moderada & $\mathrm{N}$ & 2 & 4 & 1 & \\
\hline & & $\%$ & 7,10 & 17,40 & 33,30 & \\
\hline & Severa & $\mathrm{N}$ & 1 & 4 & 2 & \\
\hline & & $\%$ & 3,60 & 17,40 & 66,70 & \\
\hline \multirow[t]{8}{*}{ Depressão } & Normal & $\mathrm{N}$ & 9 & 0 & 0 & $<0,001 *$ \\
\hline & & $\%$ & 32,10 & 0,00 & 0,00 & \\
\hline & Leve & $\mathrm{N}$ & 14 & 14 & 0 & \\
\hline & & $\%$ & 50,00 & 60,90 & 0,00 & \\
\hline & Moderada & $\mathrm{N}$ & 3 & 5 & 0 & \\
\hline & & $\%$ & 10,70 & 21,70 & 0,00 & \\
\hline & Severa & $\mathrm{N}$ & 2 & 4 & 3 & \\
\hline & & $\%$ & 7,10 & 17,40 & 100,00 & \\
\hline
\end{tabular}

Fonte: dados da pesquisa.

Nota: * indica associação significativa com sintomas da menopausa pelo teste do Qui-quadrado para $p$-valor $\leq 0,05$.

$\mathrm{Na}$ pesquisa em questão, o baixo grau de escolaridade associou-se a escores severos de ansiedade e depressão; dentre as mulheres com esses escores, $57,1 \%$ e $55,6 \%$ não completaram o ensino fundamental, respectivamente. A maioria absoluta (100\%) das participantes da pesquisa com depressão moderada e severa fazia uso regular de medicamentos $(\mathrm{p}=0,026)$, conforme Tabela 3 . 
Tabela 3 - Análise da associação da distribuição de frequências absoluta (N) e relativa (\%) da ansiedade com escolaridade, depressão com escolaridade e medicamentos. Marília, São Paulo. 2016.

\begin{tabular}{|c|c|c|c|c|c|c|c|}
\hline & & & \multicolumn{5}{|c|}{ Ansiedade } \\
\hline & & Frequência & Normal & Leve & Moderada & Severa & p-valor \\
\hline \multirow[t]{14}{*}{ Escolaridade } & Fundamental & $\mathrm{N}$ & 6 & 2 & 4 & 4 & $0,024^{*}$ \\
\hline & incompleto & $\%$ & $20,70 \%$ & $18,20 \%$ & $57,10 \%$ & $57,10 \%$ & \\
\hline & Fundamental & $\mathrm{N}$ & 5 & 1 & 1 & 1 & \\
\hline & completo & $\%$ & $17,20 \%$ & $9,10 \%$ & $14,30 \%$ & $14,30 \%$ & \\
\hline & Médio & $\mathrm{N}$ & 2 & 0 & 1 & 1 & \\
\hline & incompleto & $\%$ & $6,90 \%$ & $0,00 \%$ & $14,30 \%$ & $14,30 \%$ & \\
\hline & Médio & $\mathrm{N}$ & 11 & 6 & 0 & 1 & \\
\hline & completo & $\%$ & $37,90 \%$ & $54,50 \%$ & $0,00 \%$ & $14,30 \%$ & \\
\hline & Superior & $\mathrm{N}$ & 0 & 1 & 0 & 0 & \\
\hline & incompleto & $\%$ & $0,00 \%$ & $9,10 \%$ & $0,00 \%$ & $0,00 \%$ & \\
\hline & Superior & $\mathrm{N}$ & 5 & 1 & 1 & 0 & \\
\hline & completo & $\%$ & $17,20 \%$ & $9,10 \%$ & $14,30 \%$ & $0,00 \%$ & \\
\hline & & & \multicolumn{5}{|c|}{ Depressão } \\
\hline & & Frequência & Normal & Leve & Moderada & Severa & p-valor \\
\hline \multirow[t]{12}{*}{ Escolaridade } & Fundamental & $\mathrm{N}$ & 1 & 7 & 3 & 5 & $0,050^{*}$ \\
\hline & incompleto & $\%$ & $11,10 \%$ & $25,00 \%$ & $37,50 \%$ & $55,60 \%$ & \\
\hline & Fundamental & $\mathrm{N}$ & 2 & 4 & 1 & 1 & \\
\hline & completo & $\%$ & $22,20 \%$ & $14,30 \%$ & $12,50 \%$ & $11,10 \%$ & \\
\hline & Médio & $\mathrm{N}$ & 1 & 1 & 1 & 1 & \\
\hline & incompleto & $\%$ & $11,10 \%$ & $3,60 \%$ & $12,50 \%$ & $11,10 \%$ & \\
\hline & Médio & $\mathrm{N}$ & 3 & 12 & 2 & 1 & \\
\hline & completo & $\%$ & $33,30 \%$ & $42,90 \%$ & $25,00 \%$ & $11,10 \%$ & \\
\hline & Superior & $\mathrm{N}$ & 0 & 0 & 0 & 1 & \\
\hline & incompleto & $\%$ & $0,00 \%$ & $0,00 \%$ & $0,00 \%$ & $11,10 \%$ & \\
\hline & Superior & $\mathrm{N}$ & 2 & 4 & 1 & 0 & \\
\hline & completo & $\%$ & $22,20 \%$ & $14,30 \%$ & $12,50 \%$ & $0,00 \%$ & \\
\hline \multirow[t]{4}{*}{ Medicamentos } & Não & $\mathrm{N}$ & 3 & 5 & 0 & 0 & $0,026^{*}$ \\
\hline & & $\%$ & $33,30 \%$ & $17,90 \%$ & $0,00 \%$ & $0,00 \%$ & \\
\hline & Sim & $\mathrm{N}$ & 6 & 23 & 8 & 9 & \\
\hline & & $\%$ & $66,70 \%$ & $82,10 \%$ & $100,00 \%$ & $100,00 \%$ & \\
\hline
\end{tabular}

Fonte: dados da pesquisa.

Nota: * indica associação significativa com ansiedade pelo teste do Qui-quadrado para p-valor $\leq 0,05$. 


\section{Discussão}

O climatério ocorre entre os 39 e 65 anos. Nesse período há a menopausa, cuja idade mediana de ocorrência apresenta diferenças geográficas: dos 50,1 aos 52,8 anos na Europa; dos 50,5 aos 51,4 na América do Norte; dos 43,8 aos 53 na América Latina, próximo aos 50 anos no Brasil; e dos 42,1 aos 49,5 anos na Ásia. Assim, a precariedade do status socioeconômico está diretamente relacionada à precocidade da menopausa em asiáticas e latino-americanas e a idade média das participantes de 52,3 anos foi esperada para a região. ${ }^{(12-14)}$

Em um estudo realizado em Ouro Preto, no estado de Minas Gerais, Brasil, com 113 mulheres climatéricas, 49,6\% das entrevistadas eram casadas, predominância que se repetiu no presente estudo, correspondendo a 34 participantes $(63,0 \%)$, sendo que $79,6 \%$ do total de entrevistadas tinham filhos $(\mathrm{p}<0,001)$. $^{(15)}$

Quanto à cor, como outro dado sociodemográfico, em 2010, dentre 14 milhões de mulheres de 45 a 60 anos de idade que viviam em situação domiciliar urbana, a maioria era branca (cerca de 7,9 milhões), em relação à cor parda (5,6 milhões), preta (1,4 milhão), amarela (167,7 mil), indígena (30,2 mil) e não declarada (280). Proporção semelhante àquela encontrada no presente estudo: 36 brancas $(66,7 \%), 11$ pardas $(20,4 \%)$, cinco pretas $(9,3 \%)$ e duas de outras cores $(3,7 \%)$, com $\mathrm{p}<0,001$. Esses dados refletem a menor expectativa de vida ao nascer da população feminina negra brasileira, comparada à branca (em 2000, 69,5 anos e 73,8 anos, respectivamente), pois, embora em 2011 as negras constituíssem metade do número de mulheres do País, verificou-se que a partir dos 44 anos de idade a composição etária feminina de ambas as cores deixa de ser similar. ${ }^{(16,17)}$

Na sequência, em relação ao dado escolaridade a maioria das participantes tem o ensino médio completo e o fundamental incompleto, diferindo parcialmente da realidade brasileira, em que predomina o ensino fundamental incompleto $(29,6 \%)$ como nível de escolaridade entre as mulheres com 25 anos ou mais, e apenas $3,8 \%$ das brasileiras dessa faixa etária apresentam ensino médio incompleto em 2016. ${ }^{(18)}$ No presente estudo, o baixo grau de escolaridade associou-se aos escores severos de ansiedade e depressão.

Houve associação significativa entre os escores de depressão leve e ansiedade normal e sintomas climatéricos leves, bem como entre aquelas com sintomas fortes e ansiedade severa. Pode ter contribuído para esses resultados a semelhança entre os sintomas inqueridos nas escalas de ansiedade e depressão no climatério em relação ao IMKB. Houve também associação entre as mulheres com sintomas climatéricos fortes e depressão severa, e entre aquelas com sintomas moderados e depressão leve. Há autores que confirmam a predominância de ânimo depressivo e ansiedade de intensidades moderadas no climatério, bem como irritabilidade. ${ }^{(19)}$

Para tratar suas comorbidades, no presente estudo houve predomínio das participantes que faziam uso regular de medicamentos, assim como em uma pesquisa realizada em Ouro Preto, Minas Gerais, na qual a maioria $(60 \%)$ das suas participantes climatéricas o fazia. ${ }^{(15)} \mathrm{A}$ maioria absoluta das participantes da nossa pesquisa com depressão moderada e severa utilizava medicamentos regularmente, contudo seria necessário um estudo que identificasse quais medicamentos são utilizados e como é realizado o tratamento do transtorno depressivo apresentado pelas mulheres climatéricas para se compreender melhor essa relação.

Nos Estados Unidos, é comum o uso de medicamentos prescritos, de venda livre, fitoterápicos e de suplementos dietéticos para o tratamento de sintomas do climatério e doenças crônicas, trazendo à tona o problema das interações medicamentosas e da polifarmácia, a qual aumenta com o envelhecimento. Na literatura, relatou-se o uso comum de suplementos com anti-inflamatórios não esteroides (AINEs), hormônio tireoidiano e estrogênios. ${ }^{(20)}$ Quanto às doenças crônicas, sabe-se que em 2003, no Brasil, 29,9\% da população portava ao menos uma doença crônica, dentre elas artrite, diabetes, câncer, patologias na coluna, hipertensão arterial sistêmica, asma, cardiopatia, doença renal crônica, depressão, tuberculose, tenossinovite e cirrose. ${ }^{(21)}$ 
Outros autores destacam no Brasil a alta prevalência de hipertensão arterial sistêmica e diabetes mellitus nas mulheres menopausadas, além da contribuição do hipoestrogenismo na etiologia de doenças cardiovasculares, distúrbios do sono e osteoporose no climatério. A falta de disciplina alimentar no período também influencia o desenvolvimento de doenças cardiovasculares, contudo esse risco pode ser diminuído em $30 \%$ a $40 \%$ com a prática regular de atividade física aeróbica de moderada intensidade. ${ }^{(14,22)}$

Sono, funções cognitivas, humor, bem-estar e autoestima são beneficiados por essa atividade, que também atenua a tensão, depressão e ansiedade. Além disso, a adesão a uma dieta equilibrada, associada a exercícios físicos diários que utilizem peso, terapia de reposição hormonal e cessação do tabagismo e do etilismo abusivo são medidas que podem prevenir a osteoporose. A homeopatia pode diminuir a polifarmácia e, como a fitoterapia, melhorar a qualidade de vida da mulher climatérica ao atenuar seus sintomas, todavia faltam estudos comprobatórios com maior nível de evidência. (1,19,22-26) $^{-1}$

$\mathrm{O}$ índice menopausal refletindo sintomas climatéricos majoritariamente leves, verificado nos resultados da pesquisa, foi confirmado pela literatura. O fogacho foi o sintoma mais comum, além da irritabilidade e da ansiedade. A prevalência dos sintomas vasomotores e a sintomatologia climatérica dependem de fatores geográficos e étnicos, aparecendo os fogachos, em ordem decrescente de proporção, nas europeias (74\%), latino-americanas (45\%-69\%), norte-americanas (36\%-50\%) e asiáticas (22\%-63\%). Sintomas psíquicos no climatério, como irritabilidade, depressão, ansiedade e disfunção sexual, podem ser desencadeados por vivências negativas com as modificações biopsicossociais concomitantes à fase, e intensificados na incapacidade de readaptação e planejamento. ${ }^{(12,15,27)}$

Não obstante os sintomas climatéricos, as comorbidades e a baixa cobertura de saúde suplementar, a maior parte das mulheres inquiridas relatou ter boas condições de vida. Segundo alguns autores, a qualidade de vida no climatério é prejudicada pelo aumento da idade, comorbidades e uso de fármacos, e beneficiada pela convivência com o parceiro, que provê segurança e apoio emocional nessa fase de mudanças biopsicossociais. ${ }^{(15)}$

Houve predomínio de participantes sem cobertura de saúde suplementar na presente pesquisa, o que condiz com a realidade brasileira, pois, em 2013, 31\% das pessoas de 40 a 59 anos possuíam plano de saúde médico ou odontológico, proporção similar para ambos os sexos, com destaque para a elevada porcentagem de $36,9 \%$ na Região Sudeste. Em contrapartida, predominou no presente estudo a acessibilidade ao serviço de saúde, achado que corrobora a pesquisa do Instituto Brasileiro de Geografia e Estatística (IBGE) do ano de 2013, que investigou a realização de consulta médica no Brasil durante 12 meses, encontrando as porcentagens de $78 \%$ para as mulheres, $74,8 \%$ para as pessoas brancas e 73,5\% na faixa etária dos 40 aos 59 anos, mostrando a importância da universalidade do SUS no acesso a esses atendimentos. ${ }^{(24)}$

\section{Conclusão}

Objetivou-se identificar o perfil de mulheres climatéricas atendidas na rede básica de saúde, por meio da aplicação de escalas de ansiedade e depressão e índice menopausal. Os principais achados foram: idade média de 52,3 anos; predomínio de mulheres brancas, casadas, com filhos, ensino médio completo, acesso aos serviços de saúde, usuárias exclusivas do SUS, boas condições de saúde, uso regular de medicamentos, ansiedade ausente - segundo a respectiva escala, e escores de índice menopausal e de depressão leves. Seus principais sintomas do climatério são os fogachos, a irritabilidade e a ansiedade.

Apesar de a maioria das participantes terem completado o ensino médio, houve forte associação entre baixo grau de escolaridade e ansiedade e depressão severa. Observou-se que, de certa forma, os escores de sintomas climatéricos e depressão, e de sintomas climatéricos e ansiedade foram diretamente proporcionais; contudo, a utilização de escalas compostas por sintomas semelhantes pode ter contribuído para esses resultados. 
A partir da aplicação das escalas e do índice menopausal, concluiu-se que as mulheres climatéricas necessitam de um espaço para verbalizar seus sentimentos e dúvidas em relação ao climatério, e trocar experiências com outras mulheres. É fundamental o acesso à escuta atenta por profissionais capacitados, visando ao atendimento integral de saúde com resolutividade às necessidades da saúde feminina nessa fase do ciclo de vida. As equipes de saúde devem motivar as mulheres à prática regular de atividade física, explicando-lhes seus benefícios, e realizar a educação em saúde dessa população sobre hábitos alimentares.

Ademais, a aplicação das escalas contribuiu para o desenvolvimento das habilidades de comunicação das estudantes; construção de vínculo, por meio da relação profissional de saúde-paciente e da escuta atenta; para a prática da educação em saúde e raciocínio clínico e apreensão de conhecimentos na área da saúde feminina.

\section{Referências}

1 Ministério da Saúde (BR). Manual de atenção à mulher no climatério. Brasília (DF): Ministério da Saúde; 2008.

2 Hoffman BL, Schorge JO, Schaffer JI, Halvorson LM, Cunningham FG. Ginecologia de Williams. $2^{\mathrm{a}}$ ed. Porto Alegre: AMGH; 2014.

3 Ministério da Saúde (BR). Protocolos de atenção básica: saúde das mulheres. Brasília (DF): Ministério da Saúde; 2016.

4 Oderich CL, Wender, MCO. Climatério. Rev Bras Med. 2012; 69:2-7.

5 Instituto Brasileiro de Geografia e Estatística (IBGE). Projeção da população do Brasil e das Unidades da Federação [Internet]. Rio de Janeiro: IBGE; [citado 2021 set 6]. Disponível em: https://www.ibge.gov.br/apps/populacao/ projecao/index.html

6 Ministério da Saúde (BR). DATASUS [Internet]. 2020 [citado 2021 set 6]. Brasília, (DF): MS. Disponível em: http://tabnet.datasus.gov.br/cgi/ tabcgi.exe?popsvs/cnv/popbr.def
7 Instituto Brasileiro de Geografia e Estatística (IBGE). Mudança demográfica no Brasil no início do século XXI: subsídios para as projeções da população. Rio de Janeiro (RJ): IBGE; 2015.

8 Sousa RL, Sousa ESS, Silva JCB, Filizola RG. Fidedignidade do teste-reteste na aplicação do índice menopausal de Blatt e Kupperman. Rev Bras Ginecol Obstet [Internet]. 2000 [citado 2021 set 6]; 22(8):481-7. Disponível em: https://www.scielo.br/j/rbgo/a/SY4xGM7ZJh KVhrpnbvKjR3y/?format=pdf\&lang=pt

9 Hamilton M. The assessment of anxiety states by rating. Br J Med Psychol. 1959; 32(1):50-5. doi: https://doi.org/10.1111/j.2044-8341.1959. tb00467.x

10 Doria Filho U. Introdução à bioestatística para simples mortais. São Paulo: Negócio; 1999.

11 Mazzetto FMC. Ansiedade e depressão em mulheres climatéricas mães e em mulheres que não tiveram filhos atendidas na Rede Básica de Atenção à Saúde do Município de Marília [tese]. Botucatu (SP): Universidade Estadual Paulista "Júlio de Mesquita Filho", Faculdade de Medicina de Botucatu; 2018.

12 Palacios S, Henderson VM, Siseles N, Tan D, Villaseca P. Age of menopause and impact of climacteric symptoms by geographical region. Climacteric. 2010; 13(5):419-28. doi: 10.3109/13697137.2010.507886.

13 Freitas ER, Barbosa AJG, Reis GA, Ramada RF, Moreira LC, Gomes LB et al. Educacão em saúde para mulheres no climatério: impactos na qualidade de vida. Reprod Clim [Internet]. 2016 [citado 2018 Abr 18]; 31(1):37-43. Disponível em: https://www.sciencedirect.com/ science/article/pii/S141320871600008X

14 Santos RDS, Carvalho FGD, Lima TP, Viegas RL, Faria A, Suen VMM et al. Perfil do estado de saúde de mulheres climatéricas. Medicina (Ribeirão Preto) [Internet]. 2012 [citado 2021 ago 8]; 45(3):310-7. Disponível em: https://www.revistas.usp.br/rmrp/article/ view/47664/51404 
15 Serpa MA, Lima AA, Guimarães ACP, Carrilo MRGG, Coura-Vital W, Veloso VM. Fatores associados à qualidade de vida em mulheres no climatério. Reprod \& Clim [Internet]. 2016 [citado 2018 nov 6]; 31(2):76-81. Disponível em: https:/www.elsevier.es/es-revista-reproducaoclimaterio-385-articulo-fatores-associados-aqualidade-vida-S1413208716300152

16 Ministério da Mulher, da Família e dos Direitos Humanos (BR). Mulheres negras: dados [Internet]. 2021 [citado 2021 ago 27]. Disponível em: http://www.spm.gov.br/assuntos/ diversidade-das-mulheres/negras/dados

17 Instituto Brasileiro de Geografia e Estatística (IBGE). Sistema IBGE de recuperação automática: tabela população residente, por cor ou raça, segundo a situação do domicílio, o sexo e a idade. [citado 2021 ago 10]. Disponível em: https://sidra.ibge.gov.br/Tabela/3175\#resultado

18 Instituto Brasileiro de Geografia e Estatística (IBGE). Pesquisa Nacional por Amostra de Domicílios Contínua: Educação 2016. Rio de Janeiro (RJ): IBGE; 2017.

19 Assunção DFS, Pires DHK, Barreto, EL, Gonçalves, FA, Dias, RS. Qualidade de vida de mulheres climatéricas. Rev Soc Bras Clin Med [Internet]. 2017 [citado 2021 jun 27], 15(2): 80-3. Disponível em: http://www.sbcm.org.br/ ojs3/index.php/rsbcm/article/view/261/244

20 Gardiner P, Stargroveb MB, Dog TL. Concomitant use of prescription medications and dietary supplements in menopausal women; an approach to provider preparedness. Maturitas [Internet]. 2011 [cited 2018 May 8]; 68(3): 251-5. Available from: https://www.ncbi. nlm.nih.gov/pmc/articles/PMC3071591/pdf/ nihms256636.pdf

21 Instituto Brasileiro de Geografia e Estatística (IBGE). Indicadores Sociodemográficos e de Saúde no Brasil: 2009. Rio de Janeiro (RJ): IBGE; 2009.

22 Federação Brasileira das Associações de Ginecologia e Obstetrícia. Manual de orientação em climatério. Rio de Janeiro: FEBRASGO; 2010.
23 Sociedade Brasileira de Climatério (SOBRAC). Consenso Brasileiro Multidisciplinar de Assistência à Saúde da Mulher Climatérica. São Paulo: Segmento; 2003 [citado 2021 jun 10]. Disponível em: http://p.download.uol.com.br/ menospausa/Consenso\%20-\%20Menopausa. pdf

24 Instituto Brasileiro de Geografia e Estatística (IBGE). Pesquisa Nacional de Saúde 2013: acesso e utilização dos serviços de saúde, acidentes e violências. Rio de Janeiro (RJ): Instituto Brasileiro de Geografia e Estatística; 2015.

25 Zanette VC, Rossato AE, Citadini-Zanette V, Bernardi FBC. Prevalência do uso da fitoterapia para alívio dos sintomas apresentados em pacientes climatéricas. Arq Catarin Med [Internet]. 2011 [citado 2021 ago 8]; 40(1):12-7. Disponível em: http://www.acm.org.br/revista/ pdf/artigos/840.pdf

26 Bagnoli VR, Fonseca AM, Bagnoli F, Cezarino PYA, Silva JSP, Baracat EC. Alternativas para o tratamento não hormonal de mulheres no climatério. Rev Bras Med. 2014; 71(9):329-33.

27 Melo CRM, Reis ES, Silva LCFP, Sola EPS, Chofakian, CBN. Aplicação do índice menopausal de Kupperman: um estudo transversal com mulheres climatéricas. Espaço Saúde [Internet]. 2016 [citado 2021 ago 8]; 17(2):41-50. Disponível em: http:/www.uel.br/revistas/ uel/index.php/espacoparasaude/article/ view/25679/2
Recebido em: 15 dez. 2020

Aceito em: 20 set. 2021 
